\title{
Guidelines for Management of Treatment-Emergent Adverse Events During Rucaparib Treatment of Patients with Metastatic Castration-Resistant Prostate Cancer
}

\author{
Brian W Labadie' \\ David S Morris ${ }^{2}$ \\ Alan $\mathrm{H}$ Bryce $^{3}$ \\ Robert Given ${ }^{4,5}$ \\ Jingsong Zhang $\mathbb{D}^{6}$ \\ Wassim Abida $^{7}$ \\ Simon Chowdhury 8,9 \\ Akash Patnaik' \\ 'Section of Hematology/Oncology, \\ Department of Medicine, University of \\ Chicago, Chicago, IL, USA; ${ }^{2}$ Urology \\ Associates, P.C., Nashville, TN, USA; \\ ${ }^{3}$ Hematology and Medical Oncology, \\ Mayo Clinic, Phoenix, AZ, USA; \\ ${ }^{4}$ Department of Urology, Eastern Virginia \\ Medical School, Norfolk, VA, USA; \\ ${ }^{5}$ Urology of Virginia, Virginia Beach, VA, \\ USA; ${ }^{6}$ Genitourinary Oncology, H. Lee \\ Moffitt Cancer Center, Tampa, FL, USA; \\ ${ }^{7}$ Genitourinary Oncology Service, \\ Memorial Sloan Kettering Cancer Center, \\ New York, NY, USA; ${ }^{8}$ Medical Oncology, \\ Guy's Hospital, London, UK; 'Sarah \\ Cannon Research Institute, London, UK
}

Correspondence: Akash Patnaik Knapp Center for Biomedical Discovery, Room 7152, University of Chicago, 900

E. 57th Street, Chicago, IL, 60637

Tel +773-834-3519

Fax +773-834-0778

Email apatnaik@medicine.bsd.uchicago.

edu
Purpose: The US Food and Drug Administration has recently granted accelerated approval of the poly(ADP-ribose) polymerase (PARP) inhibitor rucaparib as treatment for men with metastatic castration-resistant prostate cancer (mCRPC) associated with a deleterious germline or somatic $B R C A 1$ or $B R C A 2$ (BRCA) alteration. As the safety profile of this new addition to the mCRPC treatment landscape may be unfamiliar to clinicians and patients, we summarize the data from the literature and provide practical guidelines for the management of treatment-emergent adverse events (TEAEs) that may occur during rucaparib treatment.

Materials and Methods: Safety data were identified from PubMed and congress publications of trials involving men with mCRPC treated with oral rucaparib monotherapy $(600 \mathrm{mg}$ twice daily). Management guidelines for TEAEs were developed based on trial protocols, prescribing information, oncology association guidance, and the authors' clinical experience. Results: In clinical trials of men with mCRPC who received rucaparib $(n=193)$, TEAEs observed were consistent with that of other PARP inhibitors. The most frequent any-grade TEAEs included gastrointestinal events, asthenia/fatigue, anemia, increased alanine/aspartate aminotransferase, rash, and thrombocytopenia; the most frequent grade $\geq 3$ TEAE was anemia. The majority of TEAEs were self-limiting and did not require treatment modification or interruption. Here, we provide recommendations on management of the most common TEAEs reported with rucaparib as well as other TEAEs of interest.

Conclusion: Rucaparib's recent approval for treatment of BRCA-mutant mCRPC is practice changing. Proper management of TEAEs will allow maximum treatment benefit for patients receiving rucaparib.

Keywords: poly(ADP-ribose) polymerase inhibitors, rucaparib, metastatic castrationresistant prostate cancer, adverse drug reaction

\section{Introduction}

Although androgen receptor (AR)-directed therapy and taxane chemotherapy can improve outcomes for men with metastatic castration-resistant prostate cancer (mCRPC), ${ }^{1-3}$ there is a critical unmet need for additional therapeutic options as most patients will eventually progress on these therapies. In tumor cells with defects in DNA homologous recombination repair (HRR) genes (eg, BRCA1, $B R C A 2, A T M, C D K 12, C H E K 2, F A N C A$ ), inhibition of poly(ADP-ribose) polymerase (PARP) leads to accumulation of DNA damage and cell death through 
a mechanism known as synthetic lethality. ${ }^{4,5}$ In metastatic prostate cancer, approximately one-third of tumors harbor mutations in BRCA1 or BRCA2 (BRCA) or other HRR genes. ${ }^{6,7}$ Small-molecule inhibitors of PARP are currently approved by the US Food and Drug Administration (FDA) for the treatment of BRCA-mutated breast and pancreatic cancers. ${ }^{8,9}$ In ovarian cancer, PARP inhibitors are approved for treatment in patients with or without BRCA or HRR gene mutations. ${ }^{8,10,11}$

In May 2020, rucaparib became the first PARP inhibitor to be approved by the FDA for the treatment of men with a deleterious BRCA alteration (germline and/or somatic)-associated mCRPC who have been treated with AR-directed therapy and a taxane-based chemotherapy. ${ }^{10}$ Regulatory approval was based on the ground breaking results from the Phase 2 TRITON2 study (NCT02952534), in which rucaparib treatment of men with $\mathrm{mCRPC}$ associated with a deleterious BRCA alteration resulted in an objective response rate of $43.5 \%$ with a median duration of response that was not reached ( $95 \%$ confidence interval, 6.4 months to not reached). ${ }^{12}$ A second PARP inhibitor, olaparib, was also approved in 2020 for men with mCRPC associated with a deleterious alteration in an HRR gene including BRCA1, BRCA2, $A T M$, and other genes. However, PARP inhibitor treatment of patients with mCRPC associated with non-BRCA HRR gene alterations has achieved less meaningful response rates. $^{13,14}$

Since PARP inhibitors have only recently been approved for the treatment of $\mathrm{mCRPC}$, clinicians and patients may not be familiar with the types of treatmentemergent adverse events (TEAEs) that may be encountered during rucaparib treatment. Here, we review the main TEAEs observed in men with mCRPC receiving rucaparib and offer guidelines on management of these TEAEs, with a focus on those which are specific to rucaparib.

\section{Methods and Search Results}

To identify publications containing safety data from clinical trials of single-agent, oral rucaparib for mCRPC treatment, we searched PubMed up to May 6, 2021, without a starting date restriction, using the following search string: "(rucaparib AND metastatic prostate cancer) NOT combination." Our PubMed search returned 18 publications. Only 4 (Anscher et al, ${ }^{15}$ Abida et al, ${ }^{12}$ Abida et al, ${ }^{14}$ and Segan et $\mathrm{al}^{16}$ ) reported clinical safety data at the recommended starting dose of oral rucaparib $600 \mathrm{mg}$ twice daily (BID), and these were included for further examination.

American Society of Clinical Oncology (ASCO) annual meeting and European Society for Medical Oncology (ESMO) congress abstracts published between January 2016 and May 2021 were also reviewed. We identified 1 presentation by Chowdhury et $\mathrm{al}^{17}$ that included additional safety-related data not available in the PubMed publications.

From the sources, we extracted the incidence of TEAEs and the proportions of patients with treatment interruptions, dose reductions, and discontinuations due to a TEAE. Per the study design, TEAEs were defined as adverse events with onset occurring on or after the first dose of rucaparib, and patients were monitored until 28 days after the last dose. ${ }^{12}$ Clovis Oncology, Inc., provided additional safety data, including the median time to first onset for TEAEs. TEAEs referred to in the manuscript are reported as preferred terms per Medical Dictionary for Regulatory Activities (MedDRA) version 20.1, and grades are based on National Cancer Institute-Common Terminology Criteria for Adverse Events version 4.03.

We identified and summarized guidelines for management of TEAEs from available sources, including the TRITON2 clinical trial protocol; the US prescribing information; publications from major oncology associations (eg, ASCO, ESMO, National Comprehensive Cancer Network); and the authors' clinical experience with patients receiving rucaparib.

\section{Rucaparib Safety Profile in Men with mCRPC}

Among men with mCRPC harboring a BRCA alteration who received rucaparib in TRITON2 $(\mathrm{n}=115), 99.1 \%$ experienced an any-grade TEAE, $60.9 \%$ experienced a grade $\geq 3$ TEAE, $63.5 \%$ had a treatment interruption or a dose reduction due to a TEAE, and $7.8 \%$ discontinued due to a TEAE. ${ }^{12}$ Mean dose intensity (actual dose received/first dose received) was 0.88 , demonstrating that patients remained on or near the 600-mg BID dose for the duration of treatment. ${ }^{12}$ The most frequent reasons for treatment interruption due to a TEAE were anemia (21.7\%), thrombocytopenia (13.9\%), and asthenia/fatigue $(9.6 \%) .{ }^{12,15}$ Similar TEAEs led to dose reduction: anemia (13.0\%), asthenia/ fatigue $(9.6 \%)$, and thrombocytopenia $(7.0 \%) .{ }^{12,15}$ One patient each $(0.9 \%)$ discontinued due to TEAEs of acute respiratory distress syndrome; increased alanine 
aminotransferase (ALT)/aspartate aminotransferase (AST); anemia; balance disorder; cardiac failure; decreased appetite, fatigue, and weight decreased; leukopenia and neutropenia; pneumonia; and prolonged QT interval. ${ }^{12,15}$

The most frequently occurring any-grade TEAEs included asthenia/fatigue, nausea, anemia, increased ALT/AST, decreased appetite, rash (combination of related preferred terms), constipation, thrombocytopenia, vomiting, and diarrhea (Table 1). ${ }^{12}$ The most frequently occurring grade $\geq 3$ TEAE was anemia $(25.2 \%)$. ${ }^{12}$ Other adverse effects of interest reported in the TRITON2 BRCA cohort included neutropenia (any grade, 10.4\%; grade $\geq 3,7.0 \%$ ) and laboratory abnormalities of decreased phosphate (any worsening shift from baseline, $67.6 \%$; maximum shift to grade $3 / 4,15.3 \%$ ) and increased creatinine (any worsening shift from baseline, $42.6 \%$; maximum shift to grade $3 / 4,1.7 \%) .{ }^{12}$ Pulmonary embolism has been reported with other PARP inhibitors in patients with $\mathrm{MCRPC}^{8}$ and was observed at a low rate among TRITON2 patients with a BRCA alteration (any grade, $2.6 \%$; grade $\geq 3,2.6 \%$ ).

For the majority of frequently reported any-grade TEAEs, the median time to first onset was within 45 days

Table I Most Frequently Occurring ( $\geq 20 \%$ ) TEAEs of Any Grade with Rucaparib in Men with mCRPC and a BRCA Alteration

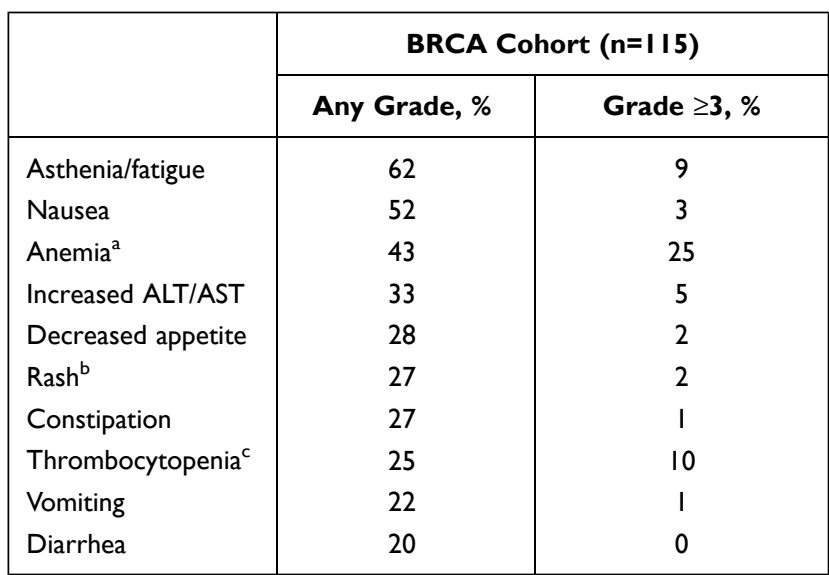

Notes: Visit cutoff date: September 13, 2019. TEAEs were graded according to $\mathrm{NCl}$ CTCAE version 4.03. There were no TEAEs of myelodysplastic syndrome or acute myeloid leukemia reported. ${ }^{a}$ Includes anemia and decreased hemoglobin. bncludes blister, blood blister, dermatitis, dermatitis contact, eczema, genital rash, palmar-plantar erythrodysesthesia syndrome, photosensitivity reaction, psoriasis, rash, rash maculo-papular, rash pruritic, skin exfoliation, skin lesion, and urticaria. Includes thrombocytopenia and platelet count decreased. Adapted from Clovis Oncology. Rubraca (Rucaparib) Tablets [Prescribing Information]. Available from: https://clovisoncology.com/pdfs/RubracaUSPI.pdf. ${ }^{10}$

Abbreviations: ALT, alanine aminotransferase; AST, aspartate aminotransferase; mCRPC, metastatic castration-resistant prostate cancer; NCI CTCAE, National Cancer Institute-Common Terminology Criteria for Adverse Events; TEAE, treatment-emergent adverse event.
(Figure 1), although anemia and thrombocytopenia had median first onset times greater than 55 days (Data on file. Clovis Oncology, Inc. 2021). Grade $\geq 3$ events for these TEAEs typically had a first onset later in treatment (Figure 1).

Rucaparib safety data reported in men with $\mathrm{MCRPC}$ and a non-BRCA HRR gene alteration $(n=78)$ were consistent with those observed in patients from TRITON2 with BRCA alterations. $^{14}$ The most frequently occurring TEAEs (all grades) reported in $\geq 20 \%$ of patients from TRITON2 with a non-BRCA HRR gene alteration were asthenia/fatigue $(52.6 \%, 41 / 78)$, nausea $(44.9 \%, 35 / 78)$, decreased appetite $(34.6 \%, 27 / 78)$, anemia $(30.8 \%, 24 / 78)$, constipation $(23.1 \%$, $18 / 78)$, vomiting $(23.1 \%, 18 / 78)$, and diarrhea $(21.8 \%, 17 /$ 78). ${ }^{14}$ The most frequently occurring grade $\geq 3$ TEAEs were anemia $(15.4 \%, 12 / 78)$, asthenia $(9.0 \%, 7 / 78)$, and thrombocytopenia $(5.1 \%, 4 / 78) .{ }^{14}$

\section{General Advice for Dosing and Delivery of Rucaparib}

The starting dose of rucaparib is $600 \mathrm{mg}$ BID. ${ }^{18}$ Patients should take two 300-mg tablets of rucaparib every 12 hours (with or without food). Missed doses (ie, not taken within 4 hours of planned time) and vomited doses should not be replaced; patients should resume dose at the next planned time. Clinicians should maintain regular clinical follow-up with patients taking rucaparib. We suggest monthly follow-up for at least the first 2 to 3 months with subsequent follow-up frequency adjusted as needed. For instance, a patient who has been tolerating rucaparib treatment well after several months may only need followup every 3 months.

Based on our experience in the TRITON2 study, we recommend performing the following clinical laboratory assessments prior to initiating rucaparib and as a part of regular follow-up: hematology - including red blood cell count and parameters, reticulocyte count, white blood cell count and differential (with absolute neutrophil count), and platelet count; serum chemistry (fasting not required) - including total protein, albumin, creatinine for estimating glomerular filtration rate (GFR) using the Cockcroft Gault formula, blood urea nitrogen or urea, total bilirubin, alkaline phosphatase, ALT, AST, lactate dehydrogenase, lipid panel, glucose, sodium, potassium, chloride, $\mathrm{CO}_{2}$, calcium, and inorganic phosphate; urinalysis - including protein, glucose, blood, $\mathrm{pH}$, and ketones. 


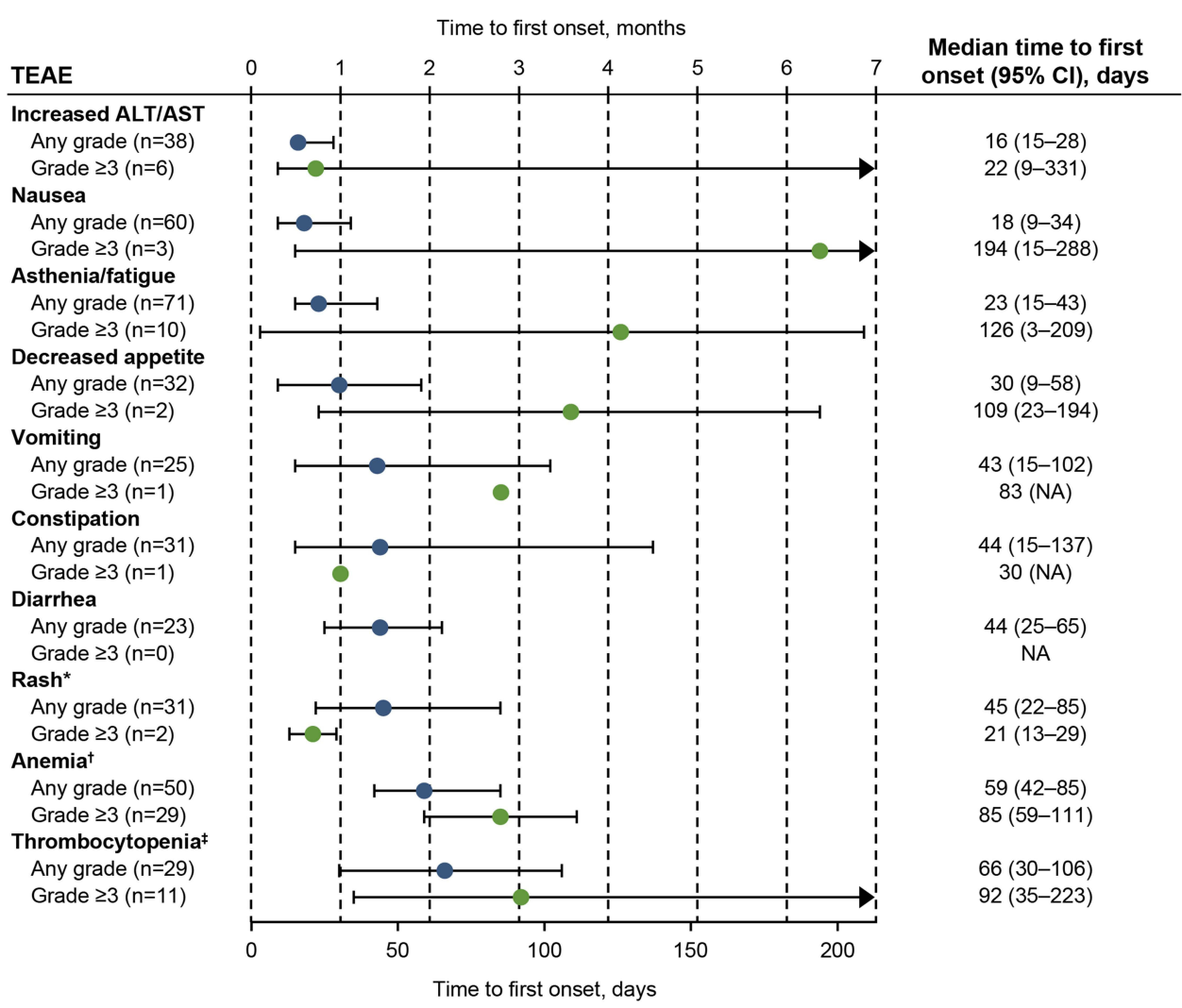

Figure I Median time to first onset for the most frequently occurring $(\geq 20 \%)$ TEAEs of any grade with rucaparib in men with mCRPC and a BRCA alteration. *Includes blister, blood blister, dermatitis, dermatitis contact, eczema, genital rash, palmar-plantar erythrodysesthesia syndrome, photosensitivity reaction, psoriasis, rash, rash maculopapular, rash pruritic, skin exfoliation, skin lesion, and urticaria. ${ }^{\dagger}$ Includes anemia and decreased hemoglobin. ${ }^{\ddagger}$ Includes thrombocytopenia and platelet count decreased. Abbreviations: mCRPC, metastatic castration-resistant prostate cancer; NA, not applicable; TEAE, treatment-emergent adverse event.

\section{Guidelines for the Management of TEAEs During Rucaparib Treatment}

Patients should be informed about the TEAEs that may occur during rucaparib treatment and how these can be monitored and managed. It would be advisable to administer rucaparib under the care of a medical oncologist specializing in treatment of advanced prostate cancer, as management of certain TEAEs will require training in internal medicine (eg, hematologic TEAEs). Per rucaparib prescribing information, adverse reactions may be managed through dose interruptions and/or dose reductions according to the severity of the reaction. ${ }^{10}$ Rucaparib dose interruption or reduction is generally advised if symptoms are not adequately controlled through other interventions. Rucaparib dose should be reduced in 100mg BID decrements using 250-mg tablets for 500-mg BID dosing and 200-mg tablets for 400-mg BID dosing.

Management recommendations for asthenia/fatigue, nausea/vomiting, decreased appetite, constipation, and diarrhea are summarized in Table 2. Online resources such as those provided by the American Cancer
Society $^{19}$ may also be helpful for patients in selfmonitoring and managing these general adverse events. Recommendations on the management of TEAEs and laboratory abnormalities that are more specific to rucaparib (eg, ALT/AST elevations, rash) or may require more a specialized approach (eg, hematologic TEAEs) are included in the sections below. These recommendations are based on prescribing information, published guidelines, and/or author experience. Local and institutional policies regarding the management of TEAEs should also be consulted.

\section{Nonhematologic TEAEs Increased ALT/AST}

Elevation in serum ALT and AST was observed in one-third of men in the BRCA cohort of TRITON2 patients. The majority of elevations in ALT and/or AST following rucaparib treatment were grades 1 or 2 , and no evidence of reduced hepatic function, such as increased bilirubin, was observed. ${ }^{12}$

Elevations in ALT and AST typically occur within the first 2-4 weeks of treatment (Figure 2A). We suggest 
Table 2 Guidelines for TEAEs Frequently Observed in Patients with mCRPC

\begin{tabular}{|c|c|c|c|c|}
\hline TEAE & \multicolumn{4}{|c|}{ Management Recommendations } \\
\hline $\begin{array}{l}\text { Asthenia/ } \\
\text { fatigue }^{4 !}\end{array}$ & \multicolumn{2}{|c|}{$\begin{array}{l}\text { Grades I-2 } \\
\text { - Implement lifestyle adjustments for } \\
\text { energy conservation and self-monitoring } \\
\text { (eg, structured daily routine, exercise } \\
\text { program tailored to limiting factors such } \\
\text { as bone metastases or fall risk) } \\
\text { - Consider nonnpharmacologic (eg, CBT) } \\
\text { interventions }\end{array}$} & \multicolumn{2}{|c|}{$\begin{array}{l}\text { Grades 3-4 } \\
\text { - Evaluate for other contributing factors (eg, pain, anemia, nausea, vomiting, } \\
\text { endocrinopathies, concomitant medications, emotional distress }{ }^{44} \text { ) and } \\
\text { manage accordingly } \\
\text { - Assess for underlying mood disorders (eg, major depressive disorder) and } \\
\text { provide pharmacologic (eg, SSRI, SNRI) or nonpharmacologic intervention as } \\
\text { needed }\end{array}$} \\
\hline $\begin{array}{l}\text { Nausea/ } \\
\text { vomiting } \\
\text { v5,46 }\end{array}$ & \multicolumn{4}{|c|}{$\begin{array}{l}\text { All grades } \\
\text { - Implement lifestyle adjustments (eg, eat small frequent meals, eat food at room temperature, take rucaparib later in the day }{ }^{47} \text { ) } \\
\text { - Consider antiemetic medications (eg, } 5-\mathrm{HT}_{3} \text { receptor antagonists, neurokinin-I antagonists, dopamine antagonists, } \\
\text { corticosteroids) if symptoms are not controlled with conservative measures } \\
\text { - Addition of or switch to another agent may be required for adequate control } \\
\text { - Dose adjustments and/or interruption may be necessary to achieve adequate control of symptoms in more severe cases }\end{array}$} \\
\hline $\begin{array}{l}\text { Decreased } \\
\text { appetite }^{\mathrm{a}}\end{array}$ & \multicolumn{4}{|c|}{$\begin{array}{l}\text { All grades } \\
\text { - Encourage patients to eat their favorite foods and have small and frequent meals }\end{array}$} \\
\hline Constipation $^{48}$ & \multicolumn{4}{|c|}{$\begin{array}{l}\text { All grades } \\
\text { - Evaluate to exclude other causes (eg, pain medication, antiemetics) } \\
\text { - Implement lifestyle adjustments (eg, increased fluid intake, maintaining activity/mobility) } \\
\text { - Use osmotic or stimulant laxatives when needed } \\
\text { - Provide suppositories and/or enemas for patients with full rectum or fecal impaction }\end{array}$} \\
\hline Diarrhea $^{49}$ & $\begin{array}{l}\text { All grades } \\
\text { - Evaluate to exclude other } \\
\text { causes }\end{array}$ & $\begin{array}{l}\text { Grades I-2 } \\
\text { - Maintain o } \\
\text { - Modify die } \\
\text { lactose) } \\
\text { - Consider F } \\
\text { antidiarrhe }\end{array}$ & $\begin{array}{l}\text { al hydration } \\
\text { (eg, avoid } \\
\text { oviding } \\
\text { I medication b }\end{array}$ & $\begin{array}{l}\text { Grades } 3-4^{c} \\
\text { - Provide antidiarrheal medication, intravenous fluids, and } \\
\text { electrolyte replacement } \\
\text { - Evaluate for infection and provide antibiotics if confirmed }\end{array}$ \\
\hline
\end{tabular}

Notes: ${ }^{a}$ Author recommendation. ${ }^{b}$ If diarrhea is not sufficiently controlled by the selected antidiarrheal medication, consider adding or replacing it with a second medication that has a different mechanism of action. ' $\mathrm{Or}$ for patients with related complications (eg, severe cramping, dehydration).

Abbreviations: 5- $\mathrm{HT}_{3}$, 5-hydroxytryptamine type 3 (serotonin); CBT, cognitive behavioral therapy; SNRI, serotonin-norepinephrine reuptake inhibitor; SSRI, selective serotonin reuptake inhibitor; TEAE, treatment-emergent adverse event.

performing laboratory screening regularly (eg, monthly) for the first 2 months of rucaparib treatment. Increases in ALT/AST of grades 1-2 can generally be monitored without treatment modification as ALT and AST levels typically normalize over time. Per the TRITON2 protocol, patients with grade 3 increases in ALT/AST and no other signs of liver dysfunction may continue rucaparib without dose modification as long as bilirubin levels are less than the upper limit of normal (ULN; Figure 3). ALT/AST levels should be monitored weekly until resolution (ie, decline to grade 2 or lower). If ALT/AST levels do not decline within 2 weeks or if they continue to increase, rucaparib should be interrupted until resolution. Rucaparib should be resumed at the same or at a reduced dosage once resolved. In our experience, for grade 4 increased
ALT/AST or elevations in bilirubin or alkaline phosphatase levels $>3 \times$ the ULN, treatment should be interrupted until resolved. Rucaparib may then be resumed at a reduced dosage, with weekly monitoring of laboratory tests for 3 weeks. If bilirubin and alkaline phosphatase levels remain elevated, we recommend evaluating for alternate etiologies of liver injury, such as hepatic metastases or viral hepatitis. In addition, consultation with a hepatologist may be considered.

\section{Cutaneous Manifestations and Photosensitivity}

Patients taking rucaparib may experience cutaneous manifestations, the most common of which were rash $(8.7 \%)$ and photosensitivity (7.0\%) (Data on file. Clovis Oncology, Inc. 2021). Rashes reported include 


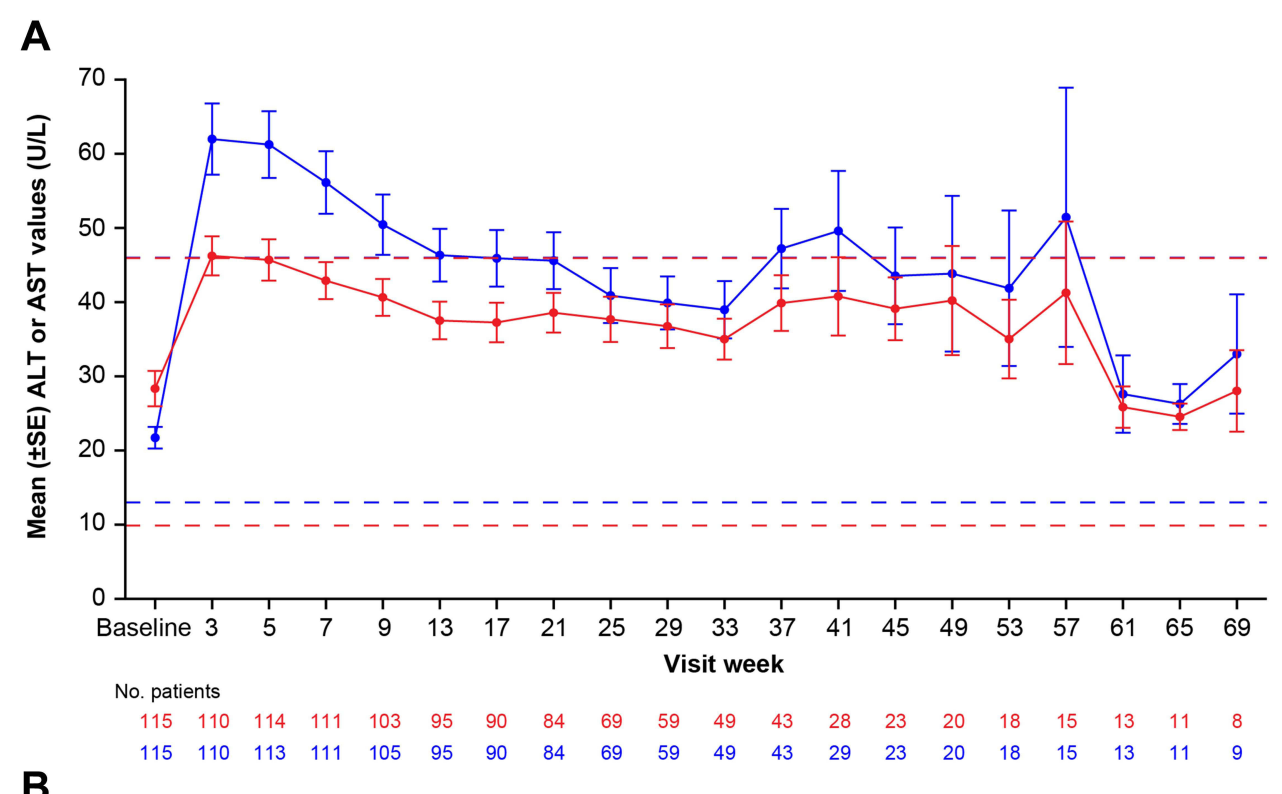

B

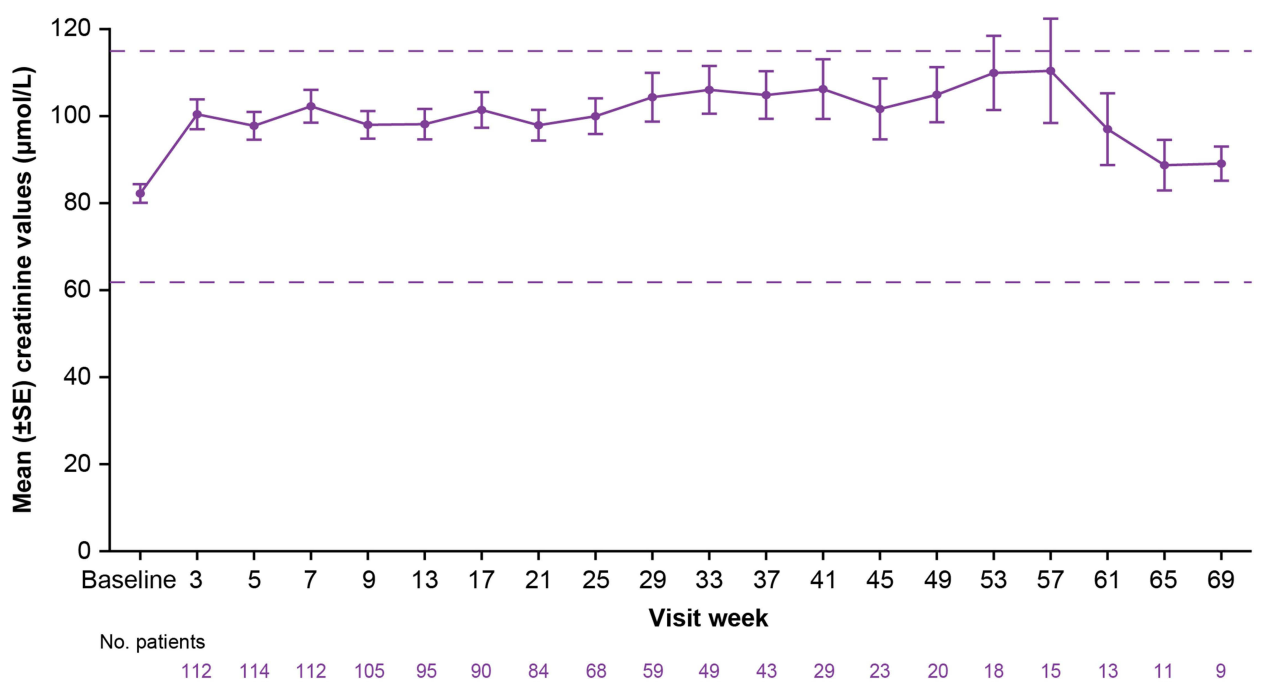

Figure 2 Mean ALT (A, blue), AST (A, red), and creatinine (B, purple) values for patients in the BRCA cohort of TRITON2. Horizontal lines represent the upper and lower limits of normal for each laboratory parameter. Error bars represent standard error of the mean.

Abbreviations: ALT, alanine aminotransferase. AST, aspartate aminotransferase. SE, standard error. Reproduced with permission from Abida W, Patnaik A, Campbell D, et al. Rucaparib in men with metastatic castration-resistant prostate cancer harboring a BRCAI or BRCA2 gene alteration. J Clin Oncol. 2020;38(32):3763-3772; Supplementary Figures SI2 and SI3, https://ascopubs.org/doi/l0.1200/JCO.20.01035 (C) Abida et al. ${ }^{12}$

eczematous, psoriatic, urticarial or pruritic eruptions, and hand-foot syndrome, for which we recommend a trial of low- to moderate-potency topical steroid (Figure 4). Urticarial eruptions and pruritus may be treated with overthe-counter antihistamines. If refractory and/or severe (eg, grade 3 or 4), early referral to a dermatologist is recommended; also consider dose interruption and/or reduction. Patients should be instructed to protect skin against excessive sun exposure (eg, wearing a hat and sunglasses using sunscreen with a sun protection factor $>50$ ) and to avoid other sources of ultraviolet light (eg, tanning beds; Figure 4).

\section{Hematologic TEAEs}

Complete blood counts should be monitored prior to treatment and monthly throughout treatment. ${ }^{10}$ Patients may have low hemoglobin at baseline due to prior therapy or due to underlying disease. ${ }^{12}$ Patients may develop anemia, thrombocytopenia, or neutropenia following initiation of rucaparib treatment (Figure 5). In our clinical experience, patients who have received prior bone-targeting radionuclide therapy (eg, Radium-223) may generally be more prone to myelosuppression.

Grade 2 anemia (hemoglobin $4.9-<6.2 \mathrm{mmol} / \mathrm{L}$ $[8-<10 \mathrm{~g} / \mathrm{dL}])$ should be evaluated for other causes, 


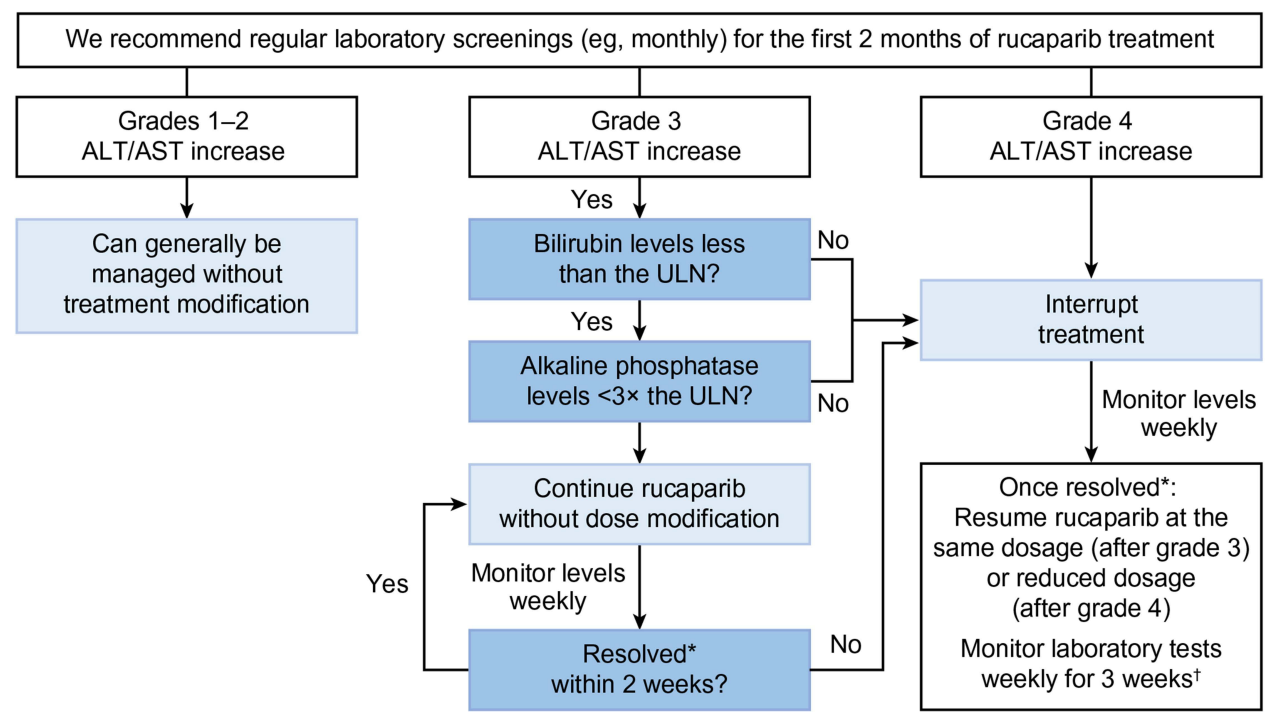

Figure 3 Guidelines for managing transaminitis. *Decline to grade 2 or lower. ${ }^{\dagger}$ If bilirubin and alkaline phosphatase levels remain elevated, we recommend evaluating for alternate etiologies of liver injury, such as hepatic metastases or viral hepatitis; consultation with a hepatologist may be considered.

Abbreviations: ALT, alanine aminotransferase; AST, aspartate aminotransferase; ULN, upper limit of normal.

\begin{tabular}{|c|c|}
\hline \multicolumn{2}{|c|}{ Patients taking rucaparib may experience rash or related skin conditions such as photosensitivity } \\
\hline$\downarrow$ & $\downarrow$ \\
\hline $\begin{array}{l}\text { Protect skin against sun exposure } \\
\text { or other sources of ultraviolet light }\end{array}$ & $\begin{array}{l}\text { Manage rash and similar conditions } \\
\text { through use of lotions or creams }\end{array}$ \\
\hline Wear a hat and clothes that cover skin & \multirow{2}{*}{$\begin{array}{l}\text { Consider use of a low- to moderate-potency } \\
\text { topical steroid (eg, hydrocortisone } 2.5 \% \text { ) } \\
\text { for eczematous or psoriatic eruptions, } \\
\text { and hand-foot syndrome* }\end{array}$} \\
\hline Use sunscreen with SPF $\geq 50$ & \\
\hline \multirow[t]{2}{*}{ Avoid tanning beds } & \\
\hline & $\begin{array}{c}\text { Provide topical steroid with or without an } \\
\text { over-the-counter antihistamine for urticarial } \\
\text { eruptions and pruritus }\end{array}$ \\
\hline
\end{tabular}

Figure 4 Guidelines for managing rash/photosensitivity. *If refractory and/or severe (eg, grade 3 or 4), early referral to a dermatologist is recommended. Abbreviation: SPF, sun protection factor.

such as iron or folate deficiency, and treated as needed (Figure 6)..$^{20,21}$ In our experience, treatment interruption may be considered for patients with grade 2 anemia. $^{20,21}$ If patients have grade $\geq 3$ anemia (hemoglobin $<4.9 \mathrm{mmol} / \mathrm{L}[<8 \mathrm{~g} / \mathrm{dL}]$ ), have related symptoms (eg, chest pain, syncope, sustained tachycardia) or have comorbidities (eg, cardiac disease), red blood cell transfusions should be used along with treatment interruption for a goal hemoglobin of $>4.3 \mathrm{mmol} / \mathrm{L}(>7 \mathrm{~g} /$ dL). ${ }^{20,21}$ Upon recovery to at least grade 2 anemia, rucaparib can be restarted at the same or lower dose.

In TRITON2, 32 (27.8\%) of 115 patients with a BRCA alteration received 1 or more transfusions of packed red blood cells. ${ }^{12}$ Decisions regarding red blood cell transfusions should be made on an individual basis and should take symptoms, comorbidities, and patient preferences into account. Hemoglobin levels should be reassessed after each transfusion. Erythropoietin, darbepoetin alfa, and/or hematopoietic colony-stimulating factors can also be considered for managing anemia; these therapies should be administered per standard of care and institutional guidelines. $^{18,19}$

Grade $\geq 2$ thrombocytopenia (platelets $<75.0 \times 10^{9} / \mathrm{L}$ ) may be managed with treatment interruption (Figure 6). Platelet transfusion should be limited to patients with active bleeding or platelets $<10 \times 10^{9} / \mathrm{L}$, which is a degree of thrombocytopenia associated with increased risk of spontaneous bleeding. ${ }^{22}$ Some medications (eg, heparin) may also cause thrombocytopenia, so concomitant medications should be reviewed. Rucaparib should be 


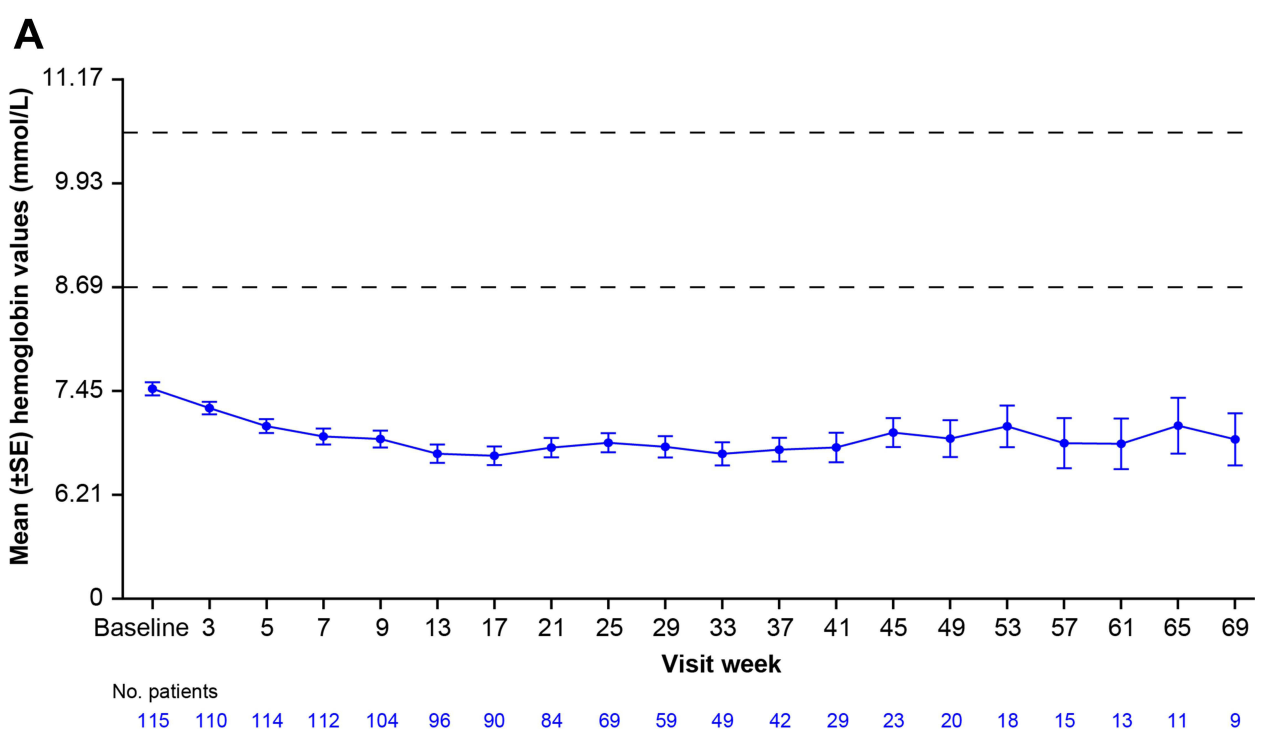

B

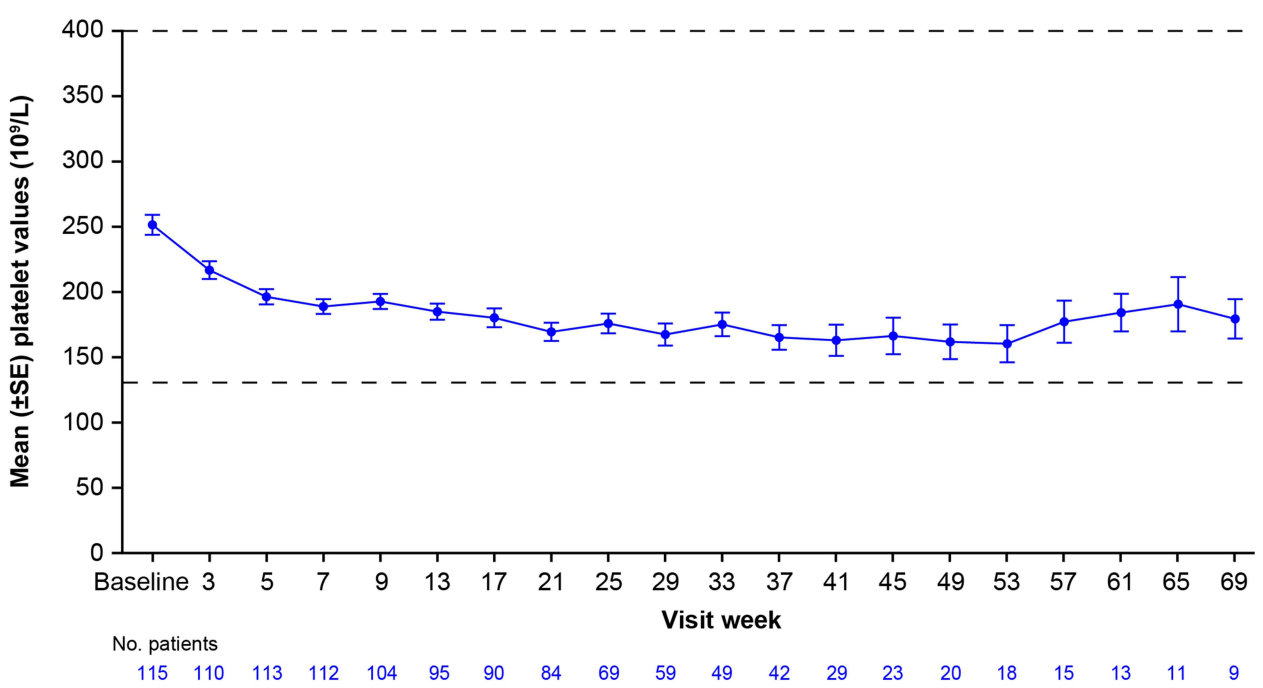

Figure 5 Mean hemoglobin (A) and platelet (B) values for patients in the BRCA cohort of TRITON2. Horizontal lines represent the upper and lower limits of normal for each laboratory parameter. Error bars represent standard error of the mean. (Data on file. Clovis Oncology, Inc. 202I).

resumed at the same or lower dose upon recovery to at least grade 1 (platelets $75.0-150 \times 10^{9} / \mathrm{L}$ ) or better.

Patients with asymptomatic grade 2 or grade 3 neutropenia (neutrophils $0.5-<1.5 \times 10^{9} / \mathrm{L}$ ) can generally continue rucaparib without dose modification (Figure 6). Use of prophylactic antibiotics in patients who experience grade 4 neutropenia without accompanying fever may vary between individual practice. Patients who experience grade 4 neutropenia and accompanying fever should be managed with hospitalization and intravenous antibiotics. Rucaparib should be held until recovery to at least grade 1 neutropenia $\left(>1.5 \times 10^{9} / \mathrm{L}\right)$ and should then be resumed at a lower dose.

\section{Other TEAEs and Laboratory} Abnormalities of Potential Interest Hypophosphatemia

Decreased laboratory values for inorganic phosphate (ie, hypophosphatemia) were identified in more than half of the patients in the TRITON2 BRCA cohort. ${ }^{12}$ Notably, most TRITON2 patients had hypophosphatemia at baseline and/or were taking medications such as antacids or bone-targeting agents, ${ }^{12}$ which are associated with decreased inorganic phosphate and are commonly used in this patient population. We recommend reviewing concomitant medications and monitoring phosphate levels prior 


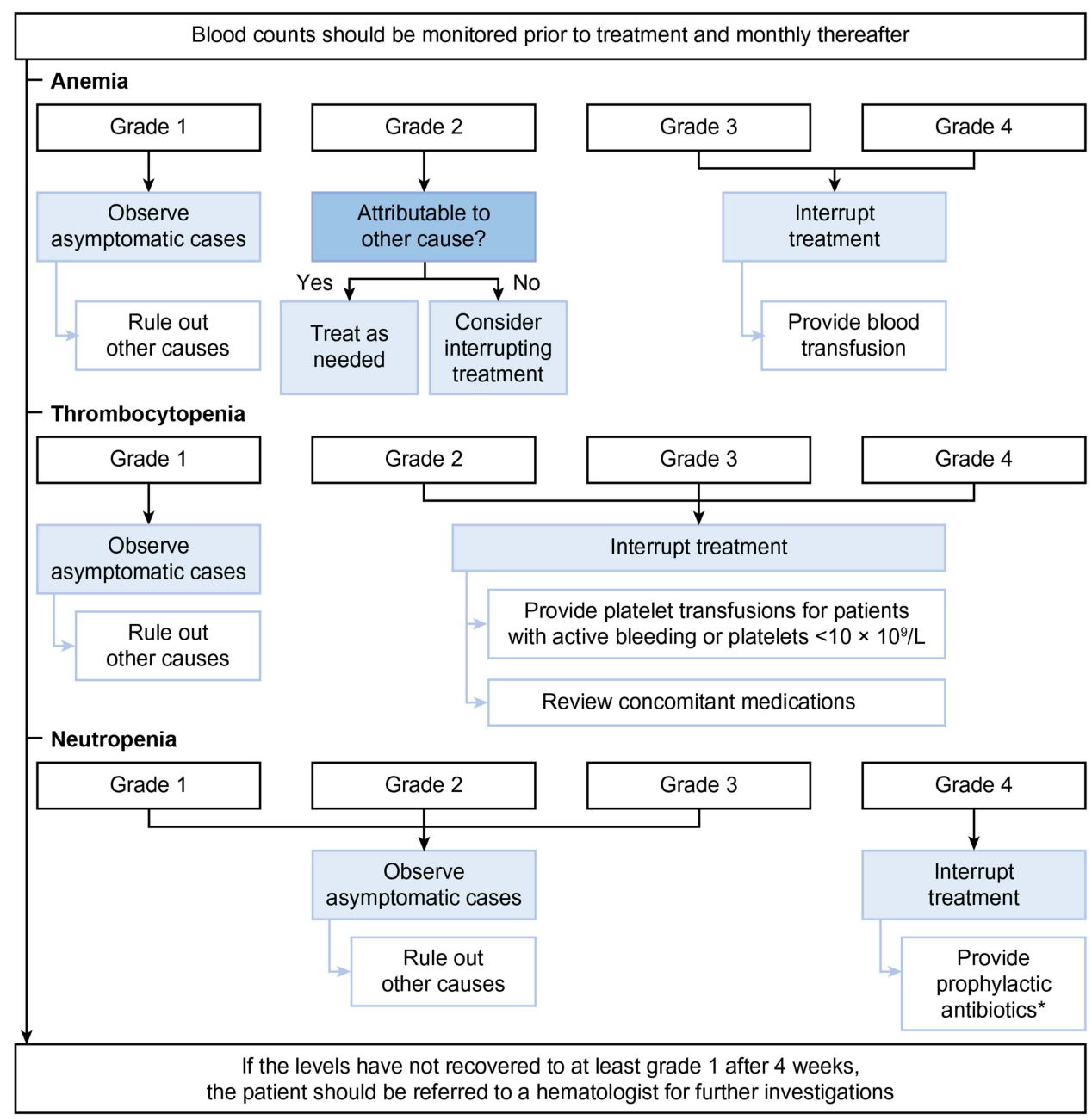

Figure 6 Guidelines for managing hematologic TEAEs.*If applicable per local guidelines. Abbreviation: TEAE, treatment-emergent adverse event.

to initiating rucaparib and monthly thereafter. Hypophosphatemia can be managed by oral phosphate repletion and routine reassessment.

\section{Increased Creatinine}

Increased creatinine levels were observed within the first 3 weeks of rucaparib treatment in TRITON2 (Figure 2B). ${ }^{12}$ PARP inhibitors have been observed to inhibit the renal transporters MATE1 and MATE2-K, which are involved in renal excretion of creatinine; thus, creatinine elevations may not reflect a true decrease in renal function. ${ }^{18,23,24}$ In a retrospective cohort of patients with endometrial or ovarian cancer treated with olaparib, rucaparib, or niraparib, discrepancies between GFRs estimated from serum creatinine levels and GFRs measured via renal scan were observed in $63 \%$ of matched assessments. ${ }^{25}$ As such, alternative methods for routine assessment of renal function in patients receiving PARP inhibitors may be needed.

Creatinine levels should be assessed prior to initiating treatment and should be monitored monthly during treatment. According to the prescribing information, dose adjustments are not necessary for patients with a mild-to-moderate reduction in GFR (creatinine clearance of $30-89 \mathrm{~mL} / \mathrm{min}$ ). ${ }^{10}$ Grade 2 serum creatinine elevations $(>1.5-3.0 \times$ baseline; $>1.5-3.0 \times$ ULN) do not require dose modification (Figure 7). For grade $\geq 3$ serum creatinine elevation $(>3.0 \times$ baseline/ULN), treatment interruption should be considered, and other pre- or post-renal causes should be evaluated (eg, dehydration, medication, ureteric/bladder outlet obstruction). An exposure-response analysis demonstrated that grade $\geq 2$ increased creatinine was correlated with a higher steady-state maximum concentration of rucaparib. ${ }^{17}$ As such, patients with true renal dysfunction may need to restart rucaparib at 


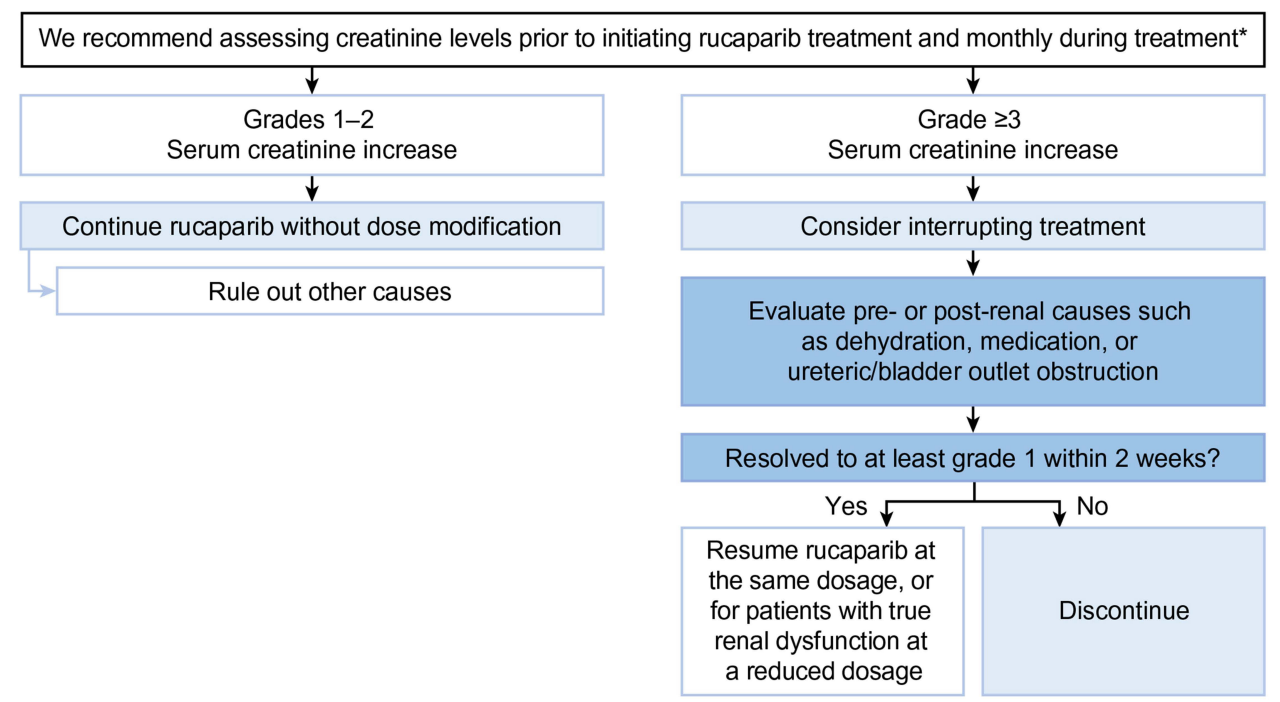

Figure 7 Guidelines for managing serum creatinine elevations. *Dose adjustments are not necessary for patients with a mild-to-moderate reduction in glomerular filtration rate (creatinine clearance of $30-89 \mathrm{~mL} / \mathrm{min}) .^{10}$

a lower dose once creatinine levels have improved. Rucaparib may need to be discontinued if toxicity does not resolve to at least grade 1 serum creatinine elevation $(>1-$ $1.5 \times$ baseline; $>\mathrm{ULN}-1.5 \times \mathrm{ULN})$ within 14 days.

\section{Pulmonary Embolism/Venous Thromboembolism}

Venous thromboembolisms (VTEs), including pulmonary embolism or deep venous thrombosis, are rare in patients receiving rucaparib. Any-grade TEAEs of VTE were reported in $0-3 \%$ of patients across various tumor types. $^{12,26,27}$ In these cases, VTE is likely related to underlying malignancy rather than to treatment. In cases of pulmonary embolism, we recommend drug interruption until the patient is clinically stable and appropriate anticoagulation treatment has been initiated.

\section{QT Prolongation}

Across studies of rucaparib in patients with various tumor types, QT prolongation has been reported in a small number of patients. ${ }^{10,28}$ Individuals with a normal baseline QT may experience a modest increase in QT interval with PARP inhibitor treatment. However, patients with pre-existing QT prolongation are at a higher risk of clinically significant prolongation. In the TRITON2 trial, 1 patient discontinued treatment due to QT prolongation. ${ }^{12}$ This patient had preexisting long QT (QTc $465 \mathrm{~ms}$ ) and demonstrated QT prolongation (QTc $589 \mathrm{~ms}) 3$ months after the initiation of rucaparib. ${ }^{16}$ Six months later, the patient was admitted to the hospital due to syncope and was found to have significant QT prolongation (QTc $680 \mathrm{~ms}$ ). Rucaparib was discontinued immediately, and isoprenaline infusion was started. The QT interval improved (QTc $547 \mathrm{~ms}$ ) within 72 hours of rucaparib cessation. The patient's health declined while in the hospital, and he died 6 days after admission with metastatic prostate cancer reported as the primary cause of death with "antecedent causes" of renal impairment and ischemic heart disease. Long QT syndrome of 5 years duration was listed as an "other significant condition" contributing to the patient's death. ${ }^{12}$

We recommend performing an electrocardiogram prior to initiating rucaparib and then monthly for the first 2 to 3 months. If there is no QTc prolongation, an EKG can be performed every 6 months for monitoring. Rucaparib should be interrupted if QTc is $>500 \mathrm{~ms}$ or prolonged by $>60 \mathrm{~ms}$ vs baseline. Rucaparib may be restarted at a lower dose once resolved. Patients should be educated on symptoms associated with QT prolongation (eg, palpitations, dyspnea, chest pain, syncope). Minimizing the use of concomitant QT-prolonging medications (eg, antiemetics, macrolide antibiotics, fluoroquinolones, selective serotonin reuptake inhibitors) is recommended.

\section{Myelodysplastic Syndrome/Acute Myeloid Lymphoma} No cases of myelodysplastic syndrome (MDS) or acute myeloid lymphoma (AML) have been reported in TRITON2 patients. ${ }^{12,14}$ Among 1146 patients who received at least 1 dose of rucaparib across clinical studies, $8(0.7 \%)$ cases of MDS/AML were reported during treatment or the 28-day safety follow-up period, with an additional $12(1.0 \%)$ cases reported among patients with long-term follow-up. ${ }^{10}$ 
Clinicians should refer patients with prolonged hematologic toxicity following dose interruption/reduction (eg, grade $\geq 2$ for $>4$ weeks) or suspected MDS/AML to a hematologist for further investigation. ${ }^{10}$ If MDS/AML is confirmed, rucaparib should be discontinued. ${ }^{10}$

\section{COVID-19}

No cases of COVID-19 have been reported in patients on the TRITON2 study (Data on file. Clovis Oncology, Inc. 2021).

\section{Discussion}

PARP inhibitors are a recent and welcome addition to the treatment of men with mCRPC. Therefore, oncologists, urologists, nurses, pharmacists and patients may be less familiar with the safety profile of these targeted agents. Here, we have provided guidelines and management recommendations for TEAEs that are observed in patients with $\mathrm{mCRPC}$ who have received rucaparib. Asthenia/fatigue, nausea/vomiting, anemia, and thrombocytopenia were the most commonly observed TEAEs that led to treatment interruption or discontinuation in studies of men with mCRPC treated with rucaparib. Anemia was the most frequent grade $\geq 3$ TEAE. The safety profile of rucaparib in men with mCRPC is similar to that observed in women with ovarian cancer, which has been well characterized in phase 2 and 3 studies. $^{27,29-31}$

When considering the use of rucaparib, patients should be informed of the specific TEAEs they may encounter and the strategies used to prevent or address TEAEs. Prompt attention to TEAEs can help reduce the need for dose interruptions or reductions or for premature discontinuation, which will ensure maximum clinical benefit. Timely and consistent management of TEAEs is likely to reduce the negative impact on patient quality of life (QoL) and enhance treatment adherence. Although there are currently no data on QoL for patients with mCRPC treated with rucaparib, future analyses of patient-reported outcomes data collected from TRITON2 and from the ongoing Phase 3 TRITON3 study (NCT02975934), which is comparing rucaparib to physician's choice of abiraterone, enzalutamide, or docetaxel as treatment for taxane-naïve patients with $\mathrm{mCRPC}$ associated with a BRCA or ATM alteration, will provide more insight on patients' experiences with treatment.

In addition to understanding which TEAEs to expect, it is useful to be aware of the timing of onset of TEAEs. The first onset of TEAEs such as increased ALT/AST, nausea, and asthenia/fatigue is typically within the first month of treatment with other TEAEs (eg, anemia, thrombocytopenia) having a later median onset. Furthermore, certain TEAEs have predictable patterns that change over the course of rucaparib treatment. For example, in the TRITON2 study, ALT, AST, and creatinine levels typically increased in the first few weeks on rucaparib and normalized over time or upon treatment discontinuation. Similar patterns have been observed in trials of rucaparib in ovarian cancer. ${ }^{27,29-31}$ Other TEAEs, such as constipation and diarrhea, are more episodic in nature, with discrete occurrences at irregular intervals. Advanced knowledge of these patterns will help patients and clinicians monitor for signs and symptoms accordingly and allow TEAEs to be detected at earlier stages.

Rucaparib is approved for use in patients with mCRPC who have progressed on AR-directed therapy (eg, abiraterone or enzalutamide) and prior taxane-based chemotherapy. As such, clinicians should take into consideration a patient's experience with TEAEs on these prior therapies as well as any disease-related symptoms that may influence their subsequent adverse event profile while receiving rucaparib treatment. Among men with $\mathrm{mCRPC}$ who receive ARdirected therapy, frequently occurring TEAEs include fatigue, hypertension, and mineralocorticoid-related events (for patients taking abiraterone). ${ }^{32-35}$ TEAEs associated with taxane in the mCRPC setting include nausea/vomiting, hematologic events, diarrhea, fatigue, and sensory/peripheral neuropathy. ${ }^{36-39}$ Disease-related symptoms may include fatigue, bone pain, and urinary frequency/urgency. ${ }^{40}$ Notably, many patients with mCRPC will have experienced fatigue due to disease and/or prior therapy. It may be helpful to discuss methods for alleviating fatigue prior to initiating rucaparib treatment, as it was the most frequently reported TEAE in TRITON2. Patients should be advised that although symptoms may be persistent/long-lasting, this does not necessarily indicate disease progression. ${ }^{41}$ Patients may also have experienced hematologic events with prior chemotherapy, and they should be informed that hematologic events observed during rucaparib treatment are typically grade 2 or less. However, any prior hematologic events should be resolved to at least grade 1 before the start of rucaparib treatment. The TRITON3 study may provide additional insight into the different AE profiles of rucaparib and current therapies.

TEAEs observed with rucaparib are consistent with those of other PARP inhibitors, ${ }^{8,9,11}$ including olaparib, which is also approved for the treatment of men with mCRPC. In particular, 
nausea, asthenia/fatigue, gastrointestinal events, and hematologic events are common in patients receiving PARP inhibitors. Additionally, some TEAEs or laboratory abnormalities of interest that occur with rucaparib have also been observed with other PARP inhibitors, including any-grade creatinine elevations ( $4 \%$ of patients with $\mathrm{mCRPC}$ receiving olaparib ${ }^{8}$ ) and any-grade pneumonitis $(2 \%$ of patients with $\mathrm{mCRPC}$ receiving olaparib ${ }^{8}$ ). Certain TEAEs have been shown to occur more frequently with other PARP inhibitors than with rucaparib, including grade $\geq 3$ thrombocytopenia ( $15 \%$ of patients with mCRPC receiving niraparib ${ }^{42}$ ). Although differences in patient populations with respect to prior treatments and disease burden may exist across the different PARP inhibitor trials, the rate of treatment discontinuation due to a TEAE was notably lower for men with $\mathrm{mCRPC}$ who received rucaparib $(5.1-7.8 \%)^{12,14}$ than for men with mCRPC who received olaparib or niraparib (18.4\% and $20.0 \%$, respectively). ${ }^{42,43}$

In conclusion, the recent FDA approval of rucaparib represents a significant advance in precision medicinebased treatment of men with mCRPC. TEAEs associated with rucaparib and other PARP inhibitors can be distinct from those encountered with chemotherapy or AR-directed therapy and require specific monitoring strategies. The ability to manage TEAEs while on rucaparib will allow patients to maintain QoL and to continue uninterrupted treatment for maximal benefit.

\section{Acknowledgments}

Medical writing and editorial assistance were provided by Nathan Yardley and Frederique H. Evans of Ashfield MedComms, an Ashfield Health company (Middletown, CT, USA), and funded by Clovis Oncology, Inc.

\section{Funding}

This work was funded by Clovis Oncology, Inc.

\section{Disclosure}

BW Labadie has nothing to disclose.

DS Morris has served in a consulting/advisory role for and received research funding from Clovis Oncology, AstraZeneca, Astellas, Bayer, Dendreon, Merck, Janssen, and Pfizer.

AH Bryce has received honoraria from Astellas and Bayer; personal fees from Merck and Castle Biosciences, outside the submitted work.

R Given has served in a consulting or advisory role and/ or on speakers bureaus for Janssen, Bayer, and Myovant and has received research funding from Clovis, Janssen, Bayer, and Merck.

J Zhang has served in a consulting or advisory role and/ or on speakers bureaus for Clovis Oncology, AstraZeneca, Bayer, Dendreon, Merck, Sanofi, and Seattle Genetics and received research funding from Astellas Pharma, AstraZeneca, and Bayer.

W Abida has served in a consulting or advisory role for Clovis Oncology, Daiichi Sankyo, Janssen Pharmaceuticals, MORE Health, and ORIC Pharmaceuticals; has received financial support for travel and/or accommodation from Clovis Oncology and ORIC Pharmaceuticals; has received personal fees from Roche, Medscape, and Onclive, outside the submitted work; and his institution has received research funding from Clovis Oncology, AstraZeneca, and Zenith Epigenetics. He is funded by National Cancer Institute (NCI) Cancer Center Support Grant P30 CA 008748, NCI Prostate Specialized Program of Research Excellence (SPORE) grant P50 CA092629-16, Department of Defense Prostate Cancer Research Program grant W81XWH-17-1-0124, and a Prostate Cancer Foundation Young Investigator Award.

S Chowdhury has served in a consulting or advisory role and/or on speakers bureaus for Clovis Oncology, Astellas, Bayer, BeiGene, Janssen, and Pfizer; received honoraria from GlaxoSmithKline; received financial support for travel and accommodation from Clovis Oncology and BeiGene: and holds stock in Curve.life; grants from Sanofi; personal fees from Huma, Remedy, Telix, Novartis, and Astra Zeneca, during the conduct of the study.

A Patnaik has served in a consulting or advisory role for Exelixis, Janssen, and Jounce Therapeutics; has received honoraria from Clovis Oncology, Merck, Prime Inc., and Roche; and has received research funding from Clovis Oncology, Progenics, Bristol Myers Squibb, and GlaxoSmithKline; and has received clinical trial support from Laekna and AstraZeneca outside the submitted work.

\section{References}

1. NCCN Clinical Practice Guidelines in Oncology: prostate cancer (Version 1.2022). Available from: https://www.nccn.org/profes sionals/physician_gls/pdf/prostate.pdf. Accessed November 18, 2021.

2. Parker C, Gillessen S, Heidenreich A, et al. Cancer of the prostate: ESMO Clinical Practice Guidelines for diagnosis, treatment and follow-up. Ann Oncol. 2015;26:v69. doi:10.1093/annonc/mdv222

3. European Association of Urology: Guidelines: prostate cancer. Available from: http://uroweb.org/guideline/prostate-cancer/. Accessed November 18, 2021.

4. Farmer H, McCabe N, Lord CJ, et al. Targeting the DNA repair defect in BRCA mutant cells as a therapeutic strategy. Nature. 2005;434:917. doi:10.1038/nature03445 
5. Ashworth A. A synthetic lethal therapeutic approach: poly(ADP) ribose polymerase inhibitors for the treatment of cancers deficient in DNA double-strand break repair. J Clin Oncol. 2008;26:3785. doi:10.1200/JCO.2008.16.0812

6. Abida W, Armenia J, Gopalan A, et al. Prospective genomic profiling of prostate cancer across disease states reveals germline and somatic alterations that may affect clinical decision making. JCO Precis Oncol. 2017;1:1.

7. Pritchard CC, Mateo J, Walsh MF, et al. Inherited DNA-repair gene mutations in men with metastatic prostate cancer. $N$ Engl $J$ Med. 2016;375:443. doi:10.1056/NEJMoa1603144

8. Lynparza (Olaparib) Tablets [Prescribing Information]. Wilmington, DE: AstraZeneca Pharmaceuticals; 2021.

9. Talzenna (Talazoparib) Capsules [Prescribing Information] New York, Ny: Pfizer, Inc; 2021.

10. Rubraca (Rucaparib) Tablets [Prescribing Information]. Boulder, CO: Clovis Oncology, Inc; 2021. Available from: https://clovisoncology. com/pdfs/RubracaUSPI.pdf. Accessed January 18, 2022.

11. Zejula (Niraparib) Capsules [Prescribing Information]. Research Triangle Park, NC: GlaxoSmithKline; 2021.

12. Abida W, Patnaik A, Campbell D, et al. Rucaparib in men with metastatic castration-resistant prostate cancer harboring a $B R C A 1$ or BRCA2 gene alteration. J Clin Oncol. 2020;38:3763. doi:10.1200/ JCO.20.01035

13. de Bono J, Mateo J, Fizazi K, et al. Olaparib for metastatic castration-resistant prostate cancer. $N$ Engl J Med. 2020;382:2091. doi:10.1056/NEJMoa1911440

14. Abida W, Campbell D, Patnaik A, et al. Non-BRCA DNA damage repair gene alterations and response to the PARP inhibitor rucaparib in metastatic castration-resistant prostate cancer: analysis from the phase 2 TRITON2 study. Clin Cancer Res. 2020;26:2487. doi:10.1158/1078-0432.CCR-20-0394

15. Anscher MS, Chang E, Gao X, et al. FDA approval summary: rucaparib for the treatment of patients with deleterious BRCA-mutated metastatic castrate-resistant prostate cancer. Oncologist. 2021;26:139. doi:10.1002/onco.13585

16. Segan L, Beekman A, Parfrey S, et al. PARP inhibitor-induced torsades de pointes in long QT syndrome: a case report. Eur Heart $J$ Case Rep. 2020;4:1. doi:10.1093/ehjcr/ytz230

17. Chowdhury S, Patnaik A, Campbell D, et al. Rucaparib population pharmacokinetics (PPK) and exposure-response (ER) analyses in patients (pts) with metastatic castration-resistant prostate cancer (mCRPC) in TRITON2. Ann Oncol. 2020;31:S533-S534. doi:10.1016/j.annonc.2020.08.918

18. Rubraca (Rucaparib) Tablets [Summary of Product Characteristics]. Swords, Ireland: Clovis Oncology Ireland Ltd; 2021.

19. American Cancer Society. Managing Cancer-related Side Effects. Available from: https://www.cancer.org/treatment/treatments-andside-effects/physical-side-effects.html. Accessed November 18, 2021.

20. NCCN. Clinical Practice Guidelines in Oncology: hematopoietic growth factors (Version 4.2021). Available from: https://www.ncen. org/professionals/physician_gls/pdf/growthfactors.pdf. Accessed November 18, 2021.

21. Aapro M, Beguin Y, Bokemeyer C, et al. Management of anaemia and iron deficiency in patients with cancer: ESMO Clinical Practice Guidelines. Ann Oncol. 2018;29:iv96. doi:10.1093/annonc/mdx758

22. Wandt H, Frank M, Ehninger G, et al. Safety and cost effectiveness of a $10 \times 10^{9} / \mathrm{L}$ trigger for prophylactic platelet transfusions compared with the traditional $20 \times 10^{9} / \mathrm{L}$ trigger: a prospective comparative trial in 105 patients with acute myeloid leukemia. Blood. 1998;91:3601. doi:10.1182/blood.V91.10.3601

23. Kikuchi R, Lao Y, Bow DA, et al. Prediction of clinical drug-drug interactions of veliparib (ABT-888) with human renal transporters (OAT1, OAT3, OCT2, MATE1, and MATE2K). J Pharm Sci. 2013;102:4426. doi:10.1002/jps.23737
24. McCormick A, Swaisland H. In vitro assessment of the roles of drug transporters in the disposition and drug-drug interaction potential of olaparib. Xenobiotica. 2017;47:903. doi:10.1080/00498254.20 16.1241449

25. Zibetti Dal Molin G, Westin SN, Msaouel P, et al. Discrepancy in calculated and measured glomerular filtration rates in patients treated with PARP inhibitors. Int $J$ Gynecol Cancer. 2020;30:89. doi:10.1136/ijgc-2019-000714

26. Swisher EM, Lin KK, Oza AM, et al. Rucaparib in relapsed, platinum-sensitive high-grade ovarian carcinoma (ARIEL2 Part 1): an international, multicentre, open-label, phase 2 trial. Lancet Oncol. 2017;18:75. doi:10.1016/S1470-2045(16)30559-9

27. Coleman RL, Oza AM, Lorusso D, et al. Rucaparib maintenance treatment for recurrent ovarian carcinoma after response to platinum therapy (ARIEL3): a randomised, double-blind, placebo-controlled, phase 3 trial. Lancet. 2017;390:1949. doi:10.1016/S0140-6736(17)32440-6

28. Food and Drug Administration (FDA): Multidisciplinary review and evaluation: rubraca (rucaparib). Available from: https://www.access $\mathrm{d} a \mathrm{ta} \cdot \mathrm{fda} \cdot \mathrm{gov} / \mathrm{drugs}$ a t fda_docs/nda/2016/ 209115Orig1s000MultiDisciplineR.pdf. Accessed November 18, 2021.

29. Ledermann JA, Oza AM, Lorusso D, et al. Rucaparib for patients with platinum-sensitive, recurrent ovarian carcinoma (ARIEL3): postprogression outcomes and updated safety from a randomised, placebo-controlled, phase 3 trial. Lancet Oncol. 2020;21:710. doi:10.1016/S1470-2045(20)30061-9

30. Kristeleit RS, Oaknin A, Ray-Coquard I, et al. Antitumor activity of the poly(ADP-ribose) polymerase inhibitor rucaparib as monotherapy in patients with platinum-sensitive, relapsed, BRCA-mutated, high-grade ovarian cancer, and an update on safety. Int $J$ Gynecol Cancer. 2019;29:1396. doi:10.1136/ijgc-2019-000623

31. Lorusso D, Garcia-Donas J, Sehouli J, et al. Management of adverse events during rucaparib treatment for relapsed ovarian cancer: a review of published studies and practical guidance. Target Oncol. 2020;15:391. doi:10.1007/s11523-020-00715-z

32. Scher HI, Fizazi K, Saad F, et al. Increased survival with enzalutamide in prostate cancer after chemotherapy. $N$ Engl $J$ Med. 2012;367:1187. doi:10.1056/NEJMoa1207506

33. Fizazi K, Scher HI, Molina A, et al. Abiraterone acetate for treatment of metastatic castration-resistant prostate cancer: final overall survival analysis of the COU-AA-301 randomised, double-blind, placebo-controlled phase 3 study. Lancet Oncol. 2012;13:983. doi:10.1016/S1470-2045(12)70379-0

34. Ryan CJ, Smith MR, de Bono JS, et al. Abiraterone in metastatic prostate cancer without previous chemotherapy. $N$ Engl $J$ Med. 2013;368:138. doi:10.1056/NEJMoa1209096

35. Beer TM, Armstrong AJ, Rathkopf DE, et al. Enzalutamide in metastatic prostate cancer before chemotherapy. $N$ Engl $J$ Med. 2014;371:424.

36. Tannock IF, de WR, Berry WR, et al. Docetaxel plus prednisone or mitoxantrone plus prednisone for advanced prostate cancer. $N$ Engl $J$ Med. 2004;351:1502. doi:10.1056/NEJMoa040720

37. Petrylak DP, Tangen CM, Hussain MHA, et al. Docetaxel and estramustine compared with mitoxantrone and prednisone for advanced refractory prostate cancer. $N$ Engl $J$ Med. 2004;351:1513. doi:10.1056/NEJMoa041318

38. de Bono JS, Oudard S, Ozguroglu M, et al. Prednisone plus cabazitaxel or mitoxantrone for metastatic castration-resistant prostate cancer progressing after docetaxel treatment: a randomised open-label trial. Lancet. 2010;376:1147. doi:10.1016/S0140-6736(10)61389-X

39. de Wit R, de Bono J, Sternberg $\mathrm{CN}$, et al. Cabazitaxel versus Abiraterone or enzalutamide in metastatic prostate cancer. $N$ Engl $J$ Med. 2019;381:2506. doi:10.1056/NEJMoa1911206

40. Holmstrom S, Naidoo S, Turnbull J, et al. Symptoms and impacts in metastatic castration-resistant prostate cancer: qualitative findings from patient and physician interviews. Patient. 2019;12:57. doi:10.1007/s40271-018-0349-x 
41. NCCN. Clinical Practice Guidelines in Oncology: cancer-related fatigue (Version 1.2021). Available from: https://www.nccn.org/pro fessionals/physician_gls/pdf/fatigue.pdf. Accessed November 18, 2021.

42. Smith MR, Sandhu SK, Kelly WK, et al. Pre-specified interim analysis of GALAHAD: a Phase II study of niraparib in patients (pts) with metastatic castration-resistant prostate cancer (mCRPC) and biallelic DNA-repair gene defects (DRD). Ann Oncol. 2019;30:abst LBA50. doi:10.1093/annonc/mdz394.043

43. Mateo J, Porta N, Bianchini D, et al. Olaparib in patients with metastatic castration-resistant prostate cancer with DNA repair gene aberrations (TOPARP-B): a multicentre, open-label, randomised, phase 2 trial. Lancet Oncol. 2020;21:162. doi:10.1016/S14702045(19)30684-9

44. Oh HS, Seo WS. Systematic review and meta-analysis of the correlates of cancer-related fatigue. Worldviews Evid Based Nurs. 2011;8:191. doi:10.1111/j.1741-6787.2011.00214.x
45. Roila F, Molassiotis A, Herrstedt J, et al. 2016 MASCC and ESMO guideline update for the prevention of chemotherapy- and radiotherapy-induced nausea and vomiting and of nausea and vomiting in advanced cancer patients. Ann Oncol. 2016;27:v119.

46. NCCN. Clinical Practice Guidelines in Oncology: antiemesis (Version 1.2021). Available from: https://www.nccn.org/professionals/physi cian_gls/pdf/antiemesis.pdf. Accessed November 18, 2021.

47. Drew Y, Kristeleit RS, Oaknin A, et al. Real-world delivery of rucaparib to patients with ovarian cancer: recommendations based on an integrated safety analysis of ARIEL2 and Study 10. Oncologist. 2020;25:e109. doi:10.1634/theoncologist.2019-0229

48. Larkin PJ, Cherny NI, La Carpia D, et al. Diagnosis, assessment and management of constipation in advanced cancer: ESMO Clinical Practice Guidelines. Ann Oncol. 2018;29:iv111. doi:10.1093/annonc/mdy148

49. Bossi P, Antonuzzo A, Cherny NI, et al. Diarrhoea in adult cancer patients: ESMO Clinical Practice Guidelines. Ann Oncol. 2018;29: iv126. doi:10.1093/annonc/mdy 145

\section{Publish your work in this journal}

Cancer Management and Research is an international, peer-reviewed open access journal focusing on cancer research and the optimal use of preventative and integrated treatment interventions to achieve improved outcomes, enhanced survival and quality of life for the cancer patient.
The manuscript management system is completely online and includes a very quick and fair peer-review system, which is all easy to use. Visit http://www.dovepress.com/testimonials.php to read real quotes from published authors. 